\title{
0 Attrakdiff-R para 0 design: uma redução do Attrakdiff para a análise holística das experiências do usuário
}

I lara Margolis

| Bernardo Providência 


\section{RESUMO}

O ser humano é um ser racional, mas também emocional. Seu inconsciente, englobando características como as emoções, as crenças e as percepções, interfere na tomada de decisão e é inerente às interações. O bem-estar, as experiências, a qualidade de vida, o engajamento e as situações emocionais são pontos tratados pelo design emocional e pelo design positivo. Todavia, a forma de captação dessas informações ainda precisa de atenção. Por sua vez, o Attrakdiff é um questionário de autorrelato, em escala de diferencial semântico, bipolar, constituído por 28 pares de palavras. Ele analisa a experiência do usuário, baseado em quatro dimensões (Atratividade, Qualidade Pragmática, Qualidade de Estimulação Hedônica e Qualidade de Identificação Hedônica). O método também pode ser encontrado na versão mini Attrakdiff, em que o questionário contempla 10 pares de palavras. Sua agilidade na aplicação quando comparado com outros métodos (como a Engenharia Kansei) é uma vantagem, mas a dificuldade em entender alguns termos e o tamanho do questionário são algumas de suas limitações. Diante disso, o presente estudo sugere o Attrakdiff-R, uma versão reduzida do original, contendo 18 pares de palavras. O método foi aplicado para 23 pessoas que trabalhavam em setores essenciais (bancária, industrial e de imprensa) e por isto estavam trabalhando presencialmente em pleno lockdown de março de 2021. O método teve consistência de acordo com o alfa de Cronbach, ele permitiu entender o usuário sob a perspectiva da experiência holística, possibilitando uma forma intermediária de abordagem. Além disso, a ferramenta converge como um possível questionário para entendimento do ser humano diante do design emocional e do design positivo..

Palavras-chave: Metodologia, Experiência do Usuário, Design Emocional. 


\section{- INTRODUÇÃO}

Em um mercado competitivo, os produtos e os serviços precisam de características e propriedades atraentes e diferenciadas para sua distinção. As emoções e os sentimentos são fatores eminentes na interação e na diferenciação destes (Hartono, Chuan \& Peacock, 2012). Técnicas e estudos de design emocional permitem estudar e monitorizar os estímulos perante o usuário, buscando o entendimento do ser humano e da sociedade em uma perspectiva mais holística do estudo das emoções, além de poder utilizar as informações para o desenvolvimento ou melhoramento de produtos, processos e ambientes, passíveis de produzir mais conforto, prazer e, consequentemente, bem-estar.

Por outro lado, é importante evidenciar a diversidade da subjetividade humana. Hancock, Pepe e Murphy (2005) exemplificam quando abordam o tema na perspectiva da estética, afirmando que ela é subjetiva e singular e, desta forma, pode desenvolver sistemas adaptáveis a culturas e mudanças pessoais, promovendo um estado contínuo de novidade e sentimento prazeroso. Para Kureemun e Fantina (2011), estes elementos de percepção têm sua própria medição e são baseados na subjetividade e no valor percebido. Para essas percepções, adicionados com as experiências e as expectativas, resultam em uma verdade estabelecida na mente do consumidor, que denominam de qualidade percebida.

O Attrakdiff é uma ferramenta desenvolvida para avaliar a opinião do usuário diante de suas experiências, da qualidade e da usabilidade de sistemas, sob a perspectiva do usuário e de potenciais usuários (RAMOS, 2016), tornando-se um método holístico de análise da experiência do usuário que se utiliza de uma escala de diferencial semântico bipolar, contendo 28 pares de palavras.

Para Oron-Gilad e Hancock (2005), o processo de design engloba as características do ser humano, o que inclui as percepções, as crenças, as motivações, as emoções, entre outros aspectos. Helander (2002), por sua vez, afirma a existência das reações afetivas do ser humano diante das suas interações. Isso é complementado por Damásio (2012) na interferência do consciente com o inconsciente, seja por emoções ou por processos cerebrais, no sistema de tomada de decisão.

Destacam-se duas áreas do design que corroboram com este tipo de análise: (1) o design emocional, que visa a lidar com situações emocionais difíceis e em como prolongar o engajamento do ser humano (Walter, 2011); e (2) o design positivo, que foca no desenvolvimento de produtos e serviços para a promoção de experiências positivas visando ao impacto e à melhoria da qualidade de vida e do bem-estar do usuário (Jimenez, Pohlmeyer \& Desmet, 2015). Todavia, Helander et al. (2015) enfatiza que as ferramentas para o estudo do design emocional ainda são imaturas. 
Em uma aplicação anterior do Attrakdiff (Margolis \& Providência, 2021), foi detectada uma insatisfação por parte dos respondentes para o tamanho do questionário, convergindo também com as limitações do questionário abordadas por Marques (2019) e Bevan et al. (2016). Perante isto, surgiu o interesse de adaptar o método do Attrakdiff para uma forma reduzida, na análise de experiência em pessoas que estavam trabalhando durante o lockdown Covid-19, ocorrido no final de março de 2021.

Portanto, este estudo visa a adaptar a ferramenta do Attrakdiff para o contexto das experiências laborais, com o objetivo de investigar o Attrakdiff Reduzido (Attrakdiff-R) como uma ferramenta de autorrelato, possibilitando, por um lado, melhor entendimento do ser humano e, por outro, contribuindo com uma metodologia para os estudos do design emocional.

\section{— SOBRE O MÉTODO ATTRAKDIFF}

O Attrakdiff foi desenvolvido por Marc Hassenzahl inicialmente para mensuração da qualidade e satisfação de softwares (Hassenzahl, Burmester \& Koller, 2003), tornou-se um método de avaliação de experiência de usuário, avaliando os aspectos pragmáticos, hedônicos e a atratividade da experiência sob quatro dimensões (Marques, 2019), sendo elas: (1) a Qualidade Pragmática (QPR), que aborda a usabilidade, funcionalidade e utilidade de um produto ou sistema, com intuito de saber se os usuários estão conseguindo atingir os objetivos com o sistema (Brennand, 2018; Ramos, 2016); (2) a Qualidade de Estimulação Hedônica (QHE), que enfatiza o bem-estar psicológico e os objetivos relacionados ao ser, ao desejo de desenvolver suas habilidades e aos conhecimentos sobre o produto (Brennand, 2018). Como também avalia a inovação, se desperta interesse ou possui recursos de interação, conteúdo e apresentação (Ramos, 2016); (3) a Qualidade de Identificação Hedônica (QHI), que analisa a identificação com o produto em um contexto social (Brennand, 2018; Ramos, 2016); e (4) a Atratividade (AT), que mensura o apelo global do produto e como afetam o julgamento como um todo (Brennand, 2018) também vendo a percepção de qualidade do usuário (Ramos, 2016).

O Attrakdiff já foi aplicado na avaliação da experiência dos usuários na área de logística (Gomes, 2017), no universo acadêmico (Ribeiro; Providência, 2020; Ribeiro, 2020; Brennand, 2018; Ramos, 2016), durante o Covid-19 (Margolis; Providência, 2021), em softwares (Marques, 2019; Oliveira, 2016). E, também, já foi analisada sob a perspectiva do design emocional em uma comparação com métodos da Engenharia Kansei, como destacado por Bevan et al. (2016). A conclusão foi que, apesar do Attrakdiff não oferecer benefícios adicionais à metodologia da Engenharia Kansei e de fornecer uma avaliação de qualidade hedônica similar, com feedbacks menos detalhados que a Engenharia Kansei, ele se torna 
mais rápido de aplicar e serve para comparar produtos e sistemas, além de permitir comparar se os projetos atendem às necessidades do usuário.

É uma ferramenta de autorrelato que, na sua forma original, utiliza um total de 28 pares de palavras opostas, em uma escala de diferencial semântico de 7 pontos, bipolar (Brennand, 2018; Marques, 2019). A análise dos resultados dá-se por meio de três formas: (1) a descrição de pares de palavras, na qual apresenta os valores médios de cada par de palavras agrupados sob as quatro dimensões; (2) o portfólio dos resultados, no qual o formato é composto por quadrantes em uma análise do QPR e do QH. Também tem sua análise sob a perspectiva da média, só que, desta vez, analisando cada uma das dimensões; e o (3) diagrama de valores médios, em que apresenta a média das quatro dimensões do produto sob as quatro dimensões. Nesta análise, a QPR indica o grau de sucesso no alcance dos objetivos, a QHI indica o nível de identificação do usuário, QHE mensura se a experiência é original, estimulante e interessante e, por fim, a AT indica o quanto o produto é atrativo (Ramos, 2016; Brennand, 2018). Ribeiro (2020) salienta a atenção na análise dos resultados, uma vez que o método se utiliza da mescla de pares de palavras invertidos e não invertidos.

Hassenzahl, Burmester e Koller (2008) sugeriram uma adaptação do método de autorrelato em forma compacta com 10 itens bipolares em uma escala de 7 pontos. Ele foi utilizado da forma como sugerido incialmente por Holl et al. (2016) em um aplicativo desenvolvido para bombeiros, por Silvennoinen, Vogel e Kujala (2014), na análise de experiências do usuário pelos elementos visuais cromáticos em aplicativos. Já Palaigeorgiou et al. (2017) utilizaram um questionário de 12 perguntas, com uma escala de 5 pontos em estudantes de uma segunda língua. $O$ interessante a perceber é que não ocorreu convergência da forma de análise.

Gomes (2017) fez um comparativo com outras metodologias de experiência do usuário e concluiu que o método Attrakdiff é viável, porém Marques (2019) aponta a dificuldade dos participantes de atender alguns termos como limitação técnica da ferramenta.

\section{- METODOLOGIA}

O Attrakdiff- $\mathrm{R}$ foi reduzido para um formato intermediário entre o Attrakdiff original e o Mini Attrakdiff. Foram utilizados 18 pares de palavras, em uma escala de 7 pontos Likert, em que foi questionado: "Os próximos pares de palavras representam fortes contrastes. Selecione a descrição que você considera mais apropriada em relação à sua experiência como profissional durante a pandemia. Sabendo que o 4 é o neutro entre as questões."

Os pares de palavras foram selecionados de acordo com o que fazia sentido no mundo empresarial, diante de uma análise dos termos já utilizados em português (Marques, 2019; Ramos, 2016) e do feedback recebido em aplicações anteriores (Ribeiro, 2020; Ribeiro, Providência, 2020; Margolis, Providência, 2021), também utilizados em português. 
A ordem dos pares de palavras, incluindo 8 termos invertidos, pode ser visualizada na Tabela 1.

Tabela 1. Apresentação dos pares de palavras - ordem realizada.

\begin{tabular}{llclll}
\hline \multicolumn{5}{c}{ Apresentação dos pares de palavras } \\
\hline 1 & Humano - Técnico & 7 & Alienador - Integrador & 13 & Ousado - Cauteloso \\
2 & Simples - Complicado & 8 & $\begin{array}{l}\text { Aproxima-me das pessoas - Afasta- } \\
\text { me das pessoas }\end{array}$ & 14 & Decepcionado - Realizado \\
3 & Profissional - Não Profissional & 9 & Não apresentável - Apresentável & 15 & Entediante - Chamativo \\
4 & Feio - Bonito & 10 & Sem imaginação - Criativo & 16 & Pouco exigente - Desafiador \\
5 & Previsível - Imprevisível & 11 & Bom - Mau & 17 & Motivador - Desencorajador \\
6 & $\begin{array}{l}\text { De baixa qualidade - De alta } \\
\text { qualidade }\end{array}$ & 12 & Confuso - Bem Estruturado & 18 & Incontrolável - Gerenciável \\
\hline
\end{tabular}

Os pares foram embaralhados e alguns opostos invertidos na apresentação do questionário, por este motivo, a Tabela 2 apresenta as palavras e sua ordem de acordo com as 4 dimensões do Attrakdiff.

Tabela 2. Pares de palavras do Attrakdiff-R.

\begin{tabular}{llll}
\multicolumn{1}{c}{ QPR } & \multicolumn{1}{c}{ QHE } & \multicolumn{1}{c}{ QHI } & \multicolumn{1}{c}{ ATT } \\
\hline Técnico - Humano & Sem imaginação - Criativo & Não profissional - Profissional & Decepcionado - Realizado \\
Complicado - Simples & Cauteloso - Ousado & Não apresentável - Apresentável & Feio - Bonito \\
Imprevisível - Previsível & Entediante - Chamativo & $\begin{array}{l}\text { De baixa qualidade - De alta } \\
\text { qualidade }\end{array}$ & Mau - Bom \\
Confuso - Bem Estruturado & Pouco exigente - Desafiador & $\begin{array}{l}\text { Alienador - Integrador } \\
\text { Incontrolável - Gerenciável }\end{array}$ & $\begin{array}{l}\text { Me aproxima das pessoas -Me } \\
\text { afasta das pessoas }\end{array}$ \\
\hline
\end{tabular}

O modelo foi aplicado para 11 pessoas do setor de Imprensa/Comunicação, para 5 pessoas do setor industrial e para 7 pessoas do setor bancário, totalizando 23 participantes.

Os participantes tinham os mais variados cargos e tempo de empresa (com funcionários com 1 ano de casa a funcionários com mais de 16 anos).

Os dados foram coletados por meio do Google Forms e tratados pelo Excel. Evidenciase que, apesar de existir um site que viabilize a pesquisa de maneira gratuita, fornecendo os resultados, o site é em inglês dentro do modelo padrão do Attrakdiff, com limite para 20 respondentes. Por estes motivos, a pesquisa foi realizada fora desta plataforma. $\mathrm{O}$ resultado foi analisado sob a perspectiva da descrição de pares de palavras e do diagrama de valores médios, dentro dos parâmetros estabelecidos pelo Attrakdiff original.

Ao término dos 18 pares de palavras, foi adicionada uma questão aberta, opcional, perguntando: "Na sua percepção, algum destes se destaca? Se quiser, pode explicar o motivo."

Para a análise da confiabilidade e consistência dos resultados do Attrakdiff- $R$, os itens invertidos foram desinvertidos e, assim, calculado o Alfa de Cronbach. Stefano et al. (2015) e Cabello e Chirinos (2012) afirmam que o valor mínimo para a consistência do alfa de Cronbach deve ser de 0,7. Freitas e Rodrigues (2005) afirmam que os limites são: (1) muito baixo para valores do alfa menor igual a 0,3 ; (2) baixa de 0,3 a menor igual a 0,6; (3) 
moderada de 0,6 a menor igual a 0,75; (4) alta de 0,75 a menor igual que 0,09 e (5) muito alta para números acima de 0,9.

\section{- RESULTADOS}

Sob a análise do diagrama dos valores médios, apresentados na Figura 1, é possível perceber que as quatro dimensões têm sua qualidade holística percebida positiva, com ativação moderada nos três setores pesquisados. Isso quer dizer que, apesar do trabalho presencial em pleno lockdown, a percepção da facilidade e funcionalidade da experiência do trabalho (QPR), o bem-estar psicológico (QHE), a identificação com o que está se fazendo (QHI) e a atratividade e a percepção holística (AT) são positivas. O setor da imprensa foi o que teve a perspectiva mais positiva, já o bancário menos positiva na comparação entre os três grupos e na análise de todos os profissionais que trabalharam durante o lockdown (denominado como 'todos' na Figura 1), o QHI foi a dimensão mais positiva, demonstrando a identificação dos profissionais com o que estavam fazendo.

Figura 1. Descrição dos pares de palavras.

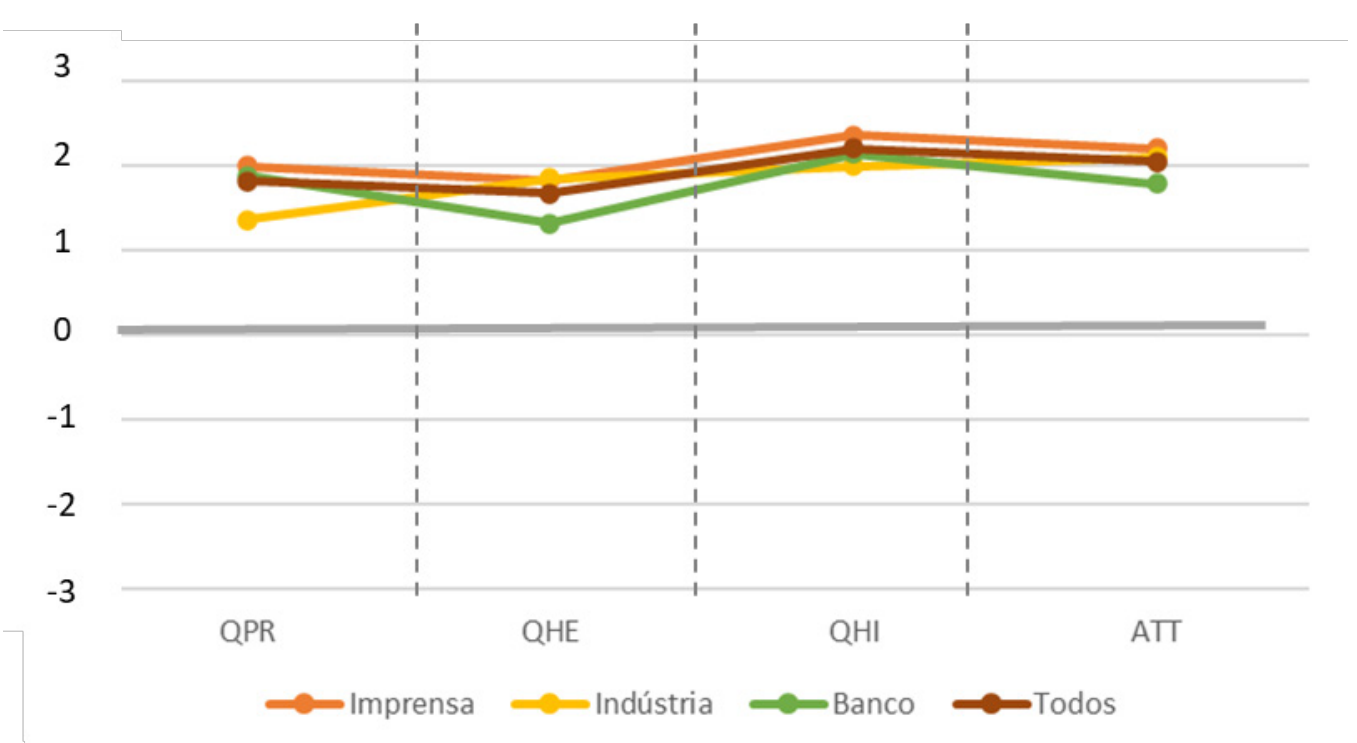

Na perspectiva da descrição dos pares de palavras, apresentado na Figura 2, de maneira geral, a experiência percebida está ativada moderadamente-leve. Entretanto, na análise comparativa, é possível perceber que há uma leve divergência entre os três grupos de análise. O setor industrial teve a percepção da experiência em mais complicada, imprevisível, de afastamento das pessoas, desafiador, de baixa qualidade, profissional, integrador e bom. Já os bancários se destacaram como humano, simples, previsível, aproxima-me das pessoas, bonito, mau e desencorajador. Por fim, as pessoas da área de imprensa tiveram o destaque em bem estruturado, gerenciável, criativo, cauteloso, chamativo, de alta qualidade, realizado e motivador. 
Todavia, se analisado na perspectiva de funcionários que trabalharam em regime presencial durante o lockdown (denominado como 'todos' na Figura 2), é possível perceber que cauteloso foi o único item negativo acionado. E profissional, desafiador, integrador $\mathrm{e}$ criativo foram os itens mais ativados.

Figura 2. Descrição dos pares de palavras.

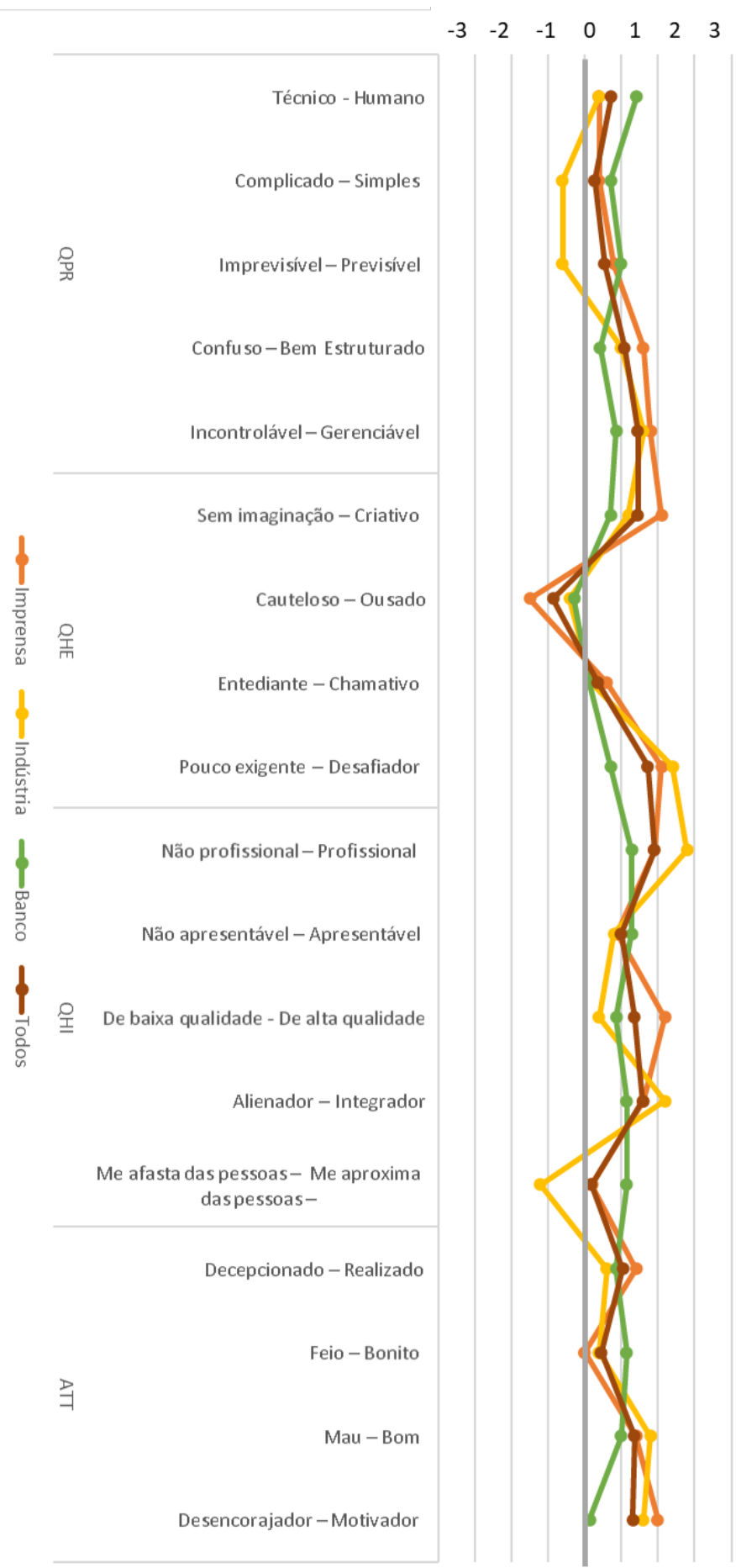

Na parte aberta do questionário, apenas uma pessoa respondeu, informando que "Afastar das pessoas se destaca. Por mais que o contato seja mantido, o distanciamento mudou a 
forma de interação entre os colegas de trabalho", o que demonstra um aspecto da pandemia (o distanciamento social) diante do momento-experiência vivenciado pelo profissional.

O alfa de Cronbach foi calculado, totalizando 0,8975 para todas as respostas, 0,8828 para os trabalhadores da imprensa, 0,8266 para os profissionais da indústria e 0,9528 para os bancários. Todos os resultados foram superiores ao mínimo de consistência do alfa de Cronbach, tendendo a uma consistência alta/muito alta, o que implica uma boa confiabilidade e consistência dos resultados.

\section{- CONSIDERAÇÕES FINAIS}

A adaptação do Attrakdiff, aqui chamado de Attrakdiff-R, adaptou o formato original do Attrakdiff para uma versão reduzida. Tendo, assim, uma opção intermediária entre o mini Attrakdiff, que utiliza 10 pares de palavras, e o original, que utiliza 28 pares de palavras. O Attrakdiff-R utilizou 18 pares de palavras, em uma escala Likert de 7 pontos, que mesclou sua apresentação entre 8 pares de palavras invertidos e 11 pares na ordem correta. O método obteve o Alfa de Cronbach geral com o valor de 0,89, o que corresponde a uma alta confiabilidade e um alto nível de consistência, demonstrando uma opção de questionário que não é tão ampla como o Attrakdiff original, nem tão minimalista como o mini Attrakdiff. Todavia, Streiner (2003) aponta que um alfa de Cronbach com valor superior a 0,90 pode indicar redundância nas respostas. Para Boyle (1991), isso não interfere na confiabilidade da escala e Peterson (1994) afirma que pode refletir boas práticas de pesquisa.

Em relação a sua aplicação, o questionário é rápido, no feedback dos participantes não foram relatadas complicações para os termos, exceto por uma pessoa que falou sobre o termo "humano-técnico". Reforça-se a atenção relatada por Ribeiro (2020) referente ao tratamento dos dados, tanto pela forma de análise dos diagramas, respeitando a sugestão de análise inicial e o uso da média, como na hora de desinverter os pares de palavras para a análise.

Por ser um questionário bipolar, o método permite entender a percepção polarizada do usuário diante de quatro perspectivas diferentes: da usabilidade e funcionalidade (QPR), do bem-estar psicológico, do desejo, da inovação e da interação (QHE), a identificação com o produto, incluindo o contexto social (QHI) e a qualidade percebida em conjunto com a atratividade (AT). Por este motivo, aborda-se aqui a ferramenta como a análise de uma experiência holística. Todavia, o questionário tem uma limitação em sua interpretação, por não permitir o entendimento da causa ou aprofundamento de algum dos itens analisados.

Para tentar resolver o problema da falta de detalhamento dos resultados, como destacado por Bevan et al. (2016), uma questão aberta opcional foi inserida, mas com apenas um respondente. A resposta refletiu o sentimento diante da pandemia, mas ainda se vê a necessidade - e oportunidade - de aperfeiçoamento deste ponto no questionário. 
Sob o resultado, foi possível perceber que, mesmo trabalhando de forma presencial em pleno lockdown, a experiência holística dos trabalhadores foi positiva, com uma leve divergência na comparação entre os três grupos de análise. O único comentário aberto retratou o sentimento de "se afastar das pessoas" diante do distanciamento social, devido ao Covid-19.

O entendimento da experiência holística converge para o entendimento do ser humano, o que pode auxiliar no desenvolvimento de melhores interações, interferindo no engajamento, nas situações emocionais e na experiência positiva. Estes fatores podem refletir na qualidade de vida e no bem-estar do usuário, além do auxílio na captação e no entendimento das percepções do usuário para maior acuracidade na tomada de decsão.. Logo, conectam-se em duas vertentes do design: (1) o design emocional e o(2) o design positivo, uma vez que auxilia na forma de entender o ser humano, organizando e estruturando os dados por meio de um questionário de autorrelato.

Entretanto, este é um estudo preliminar do Attrakdiff-R e se sugerem mais pesquisas, além do estudo com outros tipos de produtos, serviços ou ambientes para validação do método.

\section{AGRADECIMENTO}

Este trabalho foi promovido pelo Laboratório de Paisagens, Património e Território Project (Lab2PT) - UIDB/04509/2020 through FCT - Fundação para a Ciência e a Tecnologia.

\section{- REFERÊNCIAS}

1. Bevan, N., Hassenzahl, M., Liu, Z., \& Wei, W. (2016). Conparitison of Kansei Engineering and Attrakdiff to evaluate kitchen products. In: Conference Extended Abstracts on Human Factors in Computing Systems. Proceedings. San Jose: ACM, 2999-3005.

2. Brennand, C. V. L. T. (2018). Um estudo sobre a avaliação da experiência do usuário em cenários pervasivos, ubíquos e sócio-enativos. [Dissertação de Mestrado]. Departamento de Ciência da Computação. Universidade Estadual de Campinas.

3. Boyle, G. J. (1991). Does Item Homogeneity Indicate Internal Consistency or Item Redundancy in Psychometric Scales? Personality and Individual Differences, 12, 3, 291-294.

4. Cabello, E., \& Chirinos, J. L. (2012). Validación y aplicabilidad de encuestas SERVQUAL modificadas para medir la satisfacción de usuarios externos en servicios de salud. Revista Medica Herediana, 23, 2, 89-95.

5. Damásio, A. R. (2012). O erro de Descartes: Emoção, razão e cérebro humano. Companhia das Letras.

6. Freitas, A. L. P., \& Rodrigues, S. G. A. (2005). Avaliação da confiabilidade de questionário: uma análise utilizando o coeficiente alfa de Cronbach. In: Simpósio de Engenharia de Produção, 12, 07-09, Bauru-SP. Anais. Bauru-SP: UNESP. 
7. Gomes, R. V. (2017). Experiência do usuário no paradigma da internet das coisas: estudo exploratório em um sistema de gestão empresarial integrado. [Dissertação de Mestrado]. Departamento em Engenharia da Computação. Instituto de Pesquisas Tecnológicas do Estado de São Paulo.

8. Hancock, P. A., Pepe, A. A., \& Murphy, L. L. (2005). Hedonomics: The power of positive and pleasurable ergonomics. Ergonomics in design: the quarterly of human factors application, 13, 1, 8-14.

9. Hartono, M., Chuan, T. K., \& Peacock, J. B. (2012). Cultural Differences in applying Kansei Engineering to services. In: Southeast Asian Network of ergonomics Societies Conference (SEANES), 1-5, Langkawi, Quedá. Anais. Langkawi: IEEE, Curran Associates Inc.

10. Hassenzahl, M., Burmester, M., \& Koller F. (2003). AttrakDiff: Ein Fragebogen zur Messung wahrgenommener hedonischer und pragmatischer Qualität. In Mensch \& Computer 2003: Interaktion in Bewegung, G. Szwillus and J. Ziegler (Ed.). Stuttgart: B. G. Teubner, pp. 187-196.

11. Hassenzahl, M., Burmester, M., \& Koller, F. (2008). Der User Experience (UX) aufder Spur: Zum Einsatz von www.attrakdiff.de. In H. Brau, S. Diefenbach, M. Hassenzahl, F. Koller, M. Peissner \& K. Röse (Ed.), Tagungsband (pp.78-82). Stuttgart, Deutschland: Fraunhofer Verlag.

12. Helander, M. G. (2002). Hedonomics - Affective human factors design. In: Human factors and ergonomics society. Proceedings. Baltimore: SAGE Publications, 46, 978-982.

13. Helander, M., Khalid, H., Hancock, P., Jeon, M. P., Seva, R., \& Bruder, R. (2015) Affective design and EQUID: Emotional and ergonomic quality in product design and development. In: Triennial Congress of the IEA. Proceeding. Melbourne: Elsevier, 2015, p. 9-14.

14. Holl, K., Nass, C., Villela, K., \& Vieira, V. (2016). Towards a Lightweight Approach for On-site Interaction Evaluation of Safety-critical Mobile Systems. Procedia Computer Science, 94, 41-48.

15. Jimenez, S., Pohlmeyer, A., \& Desmet, P. (2015). Positive design: Reference Guide. Delft: TUDelft.

16. Kureemun, B., \& Fantina, R. (2011). Your customers' perception of quality: What it means to your bottom line and how to control it. Nova lorque: CRC Press Taylor \& Francis Group.

17. Margolis, I., \& Providência, B. (2021, in prelo). Quality Perception with Attrakdiff Method: A study in higher education during the covid-19 period. In: Martins N., Brandão D. (Ed.). Springer, 2021.

18. Marques, L. C. (2019). UX-Tips: Uma técnica de avaliação de user experiênce para aplicação de software. [Dissertação de Mestrado]. Departamento de Informática. Universidade Federal do Amazonas.

19. Oliveira, E. T. (2016). Ciberjornalismo e mobilidade: Análise da experiência do usuário de smartphone em aplicativos de noticias de referência sul-mato-grossense e nacionais [Dissertação de Mestrado]. Departamento de Comunicação. Universidade Federal de Mato Grosso do Sul.

20. Oron-Gilad, T., \& Hancock, P. A. (2005). The role of hedonomics in the future of industry, service, and product design: panel overview. In: Human factors and ergonomics society, Proceedings. Orlando: SAGE Publications, 49, 1701-1704.

21. Palaigeorgiou, G., Foteini, P., Fereniki, T., \& George, K. (2017). FingerDetectives: affordable augmented interactive miniatures for embodied vocabulary acquisition in second language learning. European Conference on Game-Based Learning, 523-531. 
22. Peterson, R. A. (1994). A meta-analysis of Cronbach's coefficient alfa. Journal of Consumer Research, 21, 2, 381-391.

23. Ramos, J. L. C. (2016). Uma abordagem preditiva da evasão na educação a distância a partir dos construtos da distância transacional. [Tese de Doutorado]. Departamento de Ciência da Computação. Universidade Federal de Pernambuco.

24. Ribeiro, I. M. (2020). Mapeamento da hedonomia e das experiências emocionais a percepção do aluno no ensino superior sob a perspectiva do design emocional. [Tese de Doutorado]. Departamento de Design. Universidade Federal de Pernambuco.

25. Ribeiro, I. M., Providência, B. (2020). Quality Perception with Attrakdiff Method: A Study in Higher Education. In: Martins N., Brandão D. (Ed.). Springer Series in Design and Innovation, 12, 222-233.

26. Silvennoinen, J., Vogel, M., \& Kujala, S. (2014). Experiencing Visual Usability and Aesthetics in Two Mobile Application Contexts. Journal of Usability Studies, 10, 1, 46-62.

27. Stefano, N. M., Casarotto Filho, N., Barichello, R., \& Sohn, A. P. (2015). A fuzzy SERVQUAL based method for ecaluated of servisse quality in the hotel industry. Procedia CIRP, 30, 433-438.

28. Streiner, D. L. (2003). Being inconsistent about consistency: when coefficient alpha does and doesn't matter. Journal of Personality Assessment, 80, 3, 217-222.

29. Walter, A. (2011) Designing for emotion. New York: A Book Apart. 